\title{
STRUCTURE AND VIBRATIONAL SPECTROSCOPIC STUDIES OF 1-NAPHTHOL: DENSITY FUNCTIONAL THEORY CALCULATIONS
}

\author{
Raja G. ${ }^{1}$, Saravanan $\mathrm{K}^{2}$ and Sivakumar $\mathbf{S}^{3}$ \\ ${ }^{1}$ Department of Chemistry, Paavai Engineering College, Namakkal-637 018, India \\ ${ }^{2}$ Department of Chemistry, Thiruvalluvar Government Arts College, Rasipuram-637 401, India \\ ${ }^{3}$ Department of Physics, Arignar Anna Government Arts College, Attur-636 121, India \\ Email: ${ }^{1}$ genuineraja@gmail.com, ${ }^{2}$ npksaran@yahoo.co.in, ${ }^{3}$ photonic_ss@ rediffmail.com
}

\begin{abstract}
The molecular vibrations of 1-Naphthol were investigated in polycrystalline sample, at room temperature, by FT- IR and FT-Raman spectroscopy. In parallel, ab initio and various density functional (DFT) methods were used to determine the geometrical, energetic and vibrational characteristics of 1-Naphthol. On the basis of B3LYP/6-31G ${ }^{*}$ and B3LYP/6-311+G ${ }^{* *}$ methods and basis set combinations, a normal mode analysis was performed to assign the various fundamental frequencies according to the total energy distribution (TED). The vibrational spectra were interpreted, with the aid of normal coordinate analysis based on a scaled quantum mechanical force field. The Infrared and Raman spectra were also predicted from the calculated intensities. Comparison of simulated spectra with the experimental spectra provides important information about the ability of the computational method to describe the vibrational modes. Simulation of Infrared and Raman spectra, utilizing the results of these calculations led to excellent overall agreement with observed spectral patterns. The investigation is performed using quantum chemical calculations conducted by means of the Gaussian 98W and Guassview set of programs. Further, density functional theory (DFT) combined with quantum chemical calculations to determine the first-order hyperpolarizability.
\end{abstract}

Keywords: Vibrational spectra; Fourier Transform infrared and FT-Raman spectra; DFT calculation, first-order hyperpolarizability

\section{INTRODUCTION}

1-Naphthol and derivatives thereof can also be used for the preparation of chiral ligands as contemplated by the present disclosure. Yet further naphthol derivatives are known in the art and are within the capacity of a skilled technician. 1-naphthol has been frequently used in chemical industries, e.g., in production of dyes, plastics, synthetic rubber, plant protecting formulations, etc. The toxicity of 1-naphthol is considered similar to that of naphthalene and carbaryl. Due to the presence of a hydroxyl group in its molecular structure, 1-naphthol solubility in water as well as its mobility in natural aquifers is enhanced. Biological monitoring is the best way for assessing exposure to organic contaminants and involves the measurement of a biomarker of exposure (usually the contaminant or a metabolite) in human blood, urine or biological tissues. So, fast, accurate and sensitive analytical methods are necessary for the examination of human exposure. 1-naphthol is an urinary metabolite of both naphthalene and carbaryl. As the biological half-live of carbaryl is on the order of days, 1-naphthol is an urinary biomarker of exposure to carbaryl indicative only of recent exposure.
Accurate vibrational assignment for aromatic and another conjugated system is necessary for characterization of materials. Assignment for complex systems can be proposed on the basis of frequency agreement between the computed harmonics and the observed fundamentals. Quantum chemical computational methods have proven to be an essential tool for interpreting and predicting vibrational spectra [1-2]. A significant advance in this area was made by scaled quantum mechanical (SQM) force field method [3-6]. In the SQM approach the systematic errors of the computed harmonic force field were corrected by a few scale factors which were found to be well transferable between chemically related molecules [2,7-9].

Recent spectroscopic studies on these materials have been motivated because the vibrational spectra are very useful for the understanding of specific biological process and in the analysis of relatively complex systems. In the present study, we extend a probing into the application of the B3LYP/6-31G ${ }^{*}$ (small basis set) and $3 \mathrm{LYP} / 6-311+\mathrm{G}^{*}$ (large basis set) based on SQM method [2] to vibrational analysis and 
conformational stability of 1-Naphthol. The main difficulty in such investigation is that the vibrational spectra of these compounds have not been completely analyzed until now and generally only rough assignments are available. The geometrical parameters of the most optimized geometry obtained via energy calculations were used for the DFT calculations. The infrared and Raman intensities were also predicted. Based on these calculations, the simulated FT-IR and FT-Raman spectra were obtained. The observed and the simulated spectra agrees well.

\section{EXPERIMENTAL DETAILS}

The fine polycrystalline samples of 1-Naphthol were obtained from the Lancaster chemical company, UK, and used as such for the spectral measurements. The room temperature Fourier transform infrared spectra of the title compounds were measured in the $4000-400 \mathrm{~cm}^{-1}$ region at a resolution $\pm 1 \mathrm{~cm}^{-1}$ using $\mathrm{KBr}$ pellets on Perkin-Elmer RX1 FT-IR spectrophotometer equipped with $\mathrm{He}-\mathrm{Ne}$ laser source, $\mathrm{KBr}$ beam splitter and $\mathrm{LiTaO} 3$ detector. Boxcar apodisation was used for the 250 averaged interferograms collected for both the samples and background. The FT-Raman spectra of 1-Naphthol were recorded on a BRUKERIFS-66V model interferometer equipped with an FRA106 and a FT-Raman accessory. The spectra were recorded in the $3500-100 \mathrm{~cm}^{-1}$ stokes region using the $1064 \mathrm{~nm}$ line of a $\mathrm{Nd}: \mathrm{YAG}$ laser for the excitation operating at $200 \mathrm{~mW}$ power. The reported wave numbers are believed to be accurate within $\pm 1 \mathrm{~cm}^{-1}$.

\section{COMPUTATIONAL DETAILS}

The calculation of the vibrational frequencies is essential and also useful for the vibrational assignments of the spectra. Quantum chemical calculations for 1-Naphthol was performed with the Gaussian $98 \mathrm{~W}$ program [10] using the Becke 3-Lee-Yang-Parr (B3LYP) functional [11,12] supplemented with the standard B3LYP/6-31G ${ }^{*}$ (small basis set) and $3 L Y P / 6-311+G^{* *}$ (large basis set) for the Cartesian representation of the theoretical force constants have been computed at the fully optimized geometry by assuming Cs point group symmetry. Scaling of the force field was performed according to the SQM procedure $[13,14]$ using selective (multiple) scaling in the natural internal coordinate representation $[15,16]$. Transformations of the force field and the subsequent normal coordinate analysis including the least squares refinement of the scaling factors, calculation of total energy distribution (TED) and IR and Raman intensities were done on a PC with the MOLVIB program (Version V7.0-G77) written by Sundius $[17,18]$.

The TED elements provide a measure of each internal coordinates contribution to the normal coordinate. For the plots of simulated IR and Raman spectra, pure Lorentzian band shapes were used with a bandwidth of $10 \mathrm{~cm}^{-1}$. The prediction of Raman intensities was carried out by following the procedure outlined below. The Raman activities calculated by the Gaussian 98 W program and adjusted during scaling procedure with MOLVIB were converted to relative Raman intensities using the following relationship derived from the basic theory of Raman scattering [19-21].

$$
l_{i}=\frac{f\left(v_{o}-v_{j}\right)^{4} S_{i}}{v_{i}\left[1-\exp \left(-h c v_{j} / k T\right)\right]}
$$

where $v_{0}$ is the exciting frequency (in $\mathrm{cm}^{-1}$ units), $v_{i}$ the vibrational wavenumber of the $i^{\text {th }}$ normal mode, $h, c$ and $k$ are the universal constants and $f$ is the suitably chosen common scaling factor for all the peak intensities.

\section{ESSENTIALS OF NONLINEAR OPTICS RELATED TO $\beta$}

The nonlinear response of an isolated molecule in an electric field $E_{i}(\omega)$ can be represented as a Taylor expansion of the total dipole moment $\mu_{t}$ induced by the field:

$$
\mu_{t}=\mu_{0}+\alpha_{i j} E_{i}+\beta_{i j k} E_{i} E_{j}+\ldots
$$

Where $\alpha$ is linear polarizability, $\mu_{0}$ the permanent dipole moment and $\beta_{i j k}$ are the first-order hyperpolarizability tensor components.

The components of first-order hyperpolarizability can be determined using the relation

$$
\beta_{i}=\beta_{i i i}+\frac{1}{3} \sum_{i \neq j}\left(\beta_{i j j}+\beta_{j i j}+\beta_{j j i}\right)
$$

Using the $x, y$ and $z$ components the magnitude of the total static dipole moment $(\mu)$, isotropic 
polarizability $\left(\alpha_{0}\right)$, first-order hyperpolarizability $\left(\beta_{\text {total }}\right)$ tensor, can be calculated by the following equations:

$$
\begin{aligned}
& \mu_{1}^{0}=\left(\mu_{x}^{2}+\mu_{y}^{2}+\mu_{z}^{2}\right)^{1 / 2} \\
& \beta_{\text {tot }}=\left(\beta_{x}^{2}+\beta_{y}^{2}\right)^{1 / 2}
\end{aligned}
$$

The complete equation for calculating the first-order hyperpolarizability from Gaussian $98 \mathrm{~W}$ output is given as follows [10]:

$$
\begin{aligned}
\beta_{\text {tot }}=\left[\left(\beta_{x x x}+\beta_{x y y}+\beta_{x z z}\right)^{2}+\left(\beta_{y y y}+\beta_{y z z}\right.\right. \\
\left.\left.+\beta_{y x x}\right)^{2}+\left(\beta_{z z z}+\beta_{z x x}+\beta_{z y y}\right)^{2}\right]
\end{aligned}
$$

The $\beta$ components of GAUSSIAN 98 W output are reported in atomic units, the calculated values have to be converted into electrostatic units $(1 \mathrm{a} . \mathrm{u}=8.3693$ $\left.\times 10^{-33} \mathrm{esu}\right)$.

Before calculating the hyperpolarizability for the in-vestigated compound, the optimization has been carried out in the UHF (unrestricted open-shell Hartree-Fock) level. Molecular geometries were fully optimized by Berny's optimization algorithm using redun-dant internal coordinates. All optimized structures were confirmed to be minimum energy conformations.

An optimization is complete when it has converged. i.e., when it has reached a minimum on the potential energy surface, thereby predicting the equilibrium structures of the molecules. This criterion is very important in geometry optimization. The inclusion of $d$ polarization and double zeta function in the split valence basis set is expected to produce a marked improvement in the calculated geometry [22]. At the optimized structure, no imaginary frequency modes were obtained proving that a true minimum on the potential energy surface was found. The electric dipole moment and dispersion free first-order hyperpolarizibility were calculated using finite field method. The finite field method offers a straight forward approach to the calcula-tion of hyperpolarizabilities [23]. All the calculations were carried out at the DFT level using the three-parameter hybrid density functional B3LYP and a 3-21 $G(d, p)$ basis set.

\section{RESULTS AND DISCUSSION}

\section{A. Molecular Geometry}

The optimized molecular structure of 1-Naphthol was shown in Fig. 1. The global minimum energy obtained by the DFT structure optimization was presented in Table 1. The optimized geometrical parameters obtained by the large basis set calculation were presented in Table 2.

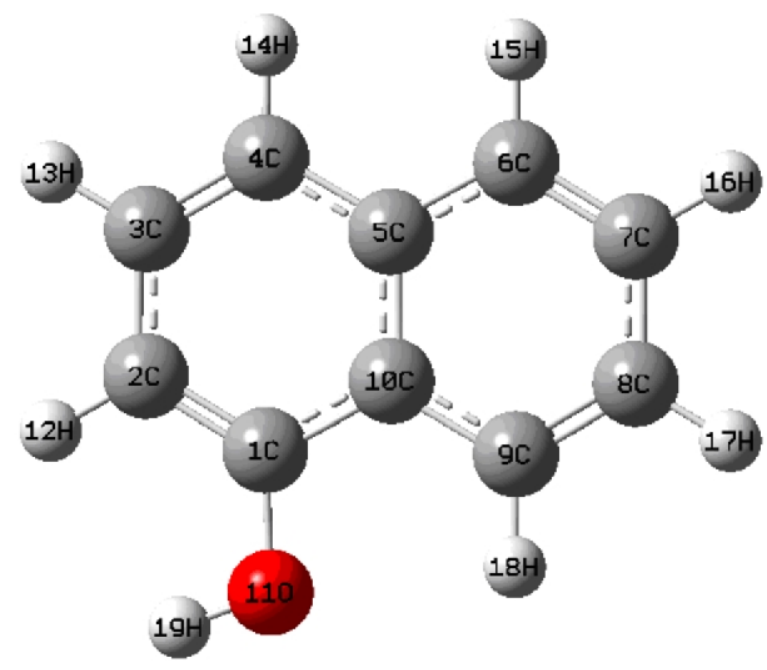

Fig. 1. Optimized molecular structure of 1-Naphthol

Table 1. Total energies of 1-Naphthol, Calculated at DFT (B3LYP)/6-31G and (B3LYP)/6-311+G level

\begin{tabular}{|c|c|}
\hline Method & Energies (Hartrees) \\
\hline $6-31 \mathrm{G}^{*}$ & -461.084753 \\
\hline $6-311+\mathrm{G}^{*}$ & -461.109185 \\
\hline
\end{tabular}

Detailed description of vibrational modes can be given by means of normal coordinate analysis (NCA). For this purpose, the full set of 72 standard internal coordinates containing 21 redundancies were defined as given in Table 3. From these, a non-redundant set of local symmetry coordinates were constructed by suitable linear combinations of internal coordinates following the recommendations of Fogarasi et. al [15, 16] are summarized in Table 4. The theoretically calculated DFT force fields were transformed in this later set of vibrational coordinates and used in all subsequent calculations. 
Table 2. Optimized geometrical parameters of 1-Naphthol obtained by B3LYP/ $6-311+G$ density functional calculations

\begin{tabular}{|c|c|c|c|c|c|}
\hline Bond length & Value $(\AA)$ & Bond angle & Value $(\AA ̊ \cap)$ & Dihedral angle & Value $(\AA)$ \\
\hline $\mathrm{C} 2-\mathrm{C} 1$ & 1.379 & C3-C2-C1 & 120.157 & C4-C3-C2-C1 & -0.013 \\
\hline C3-C2 & 1.414 & C4-C3-C2 & 120.797 & C5-C4-C3-C2 & 0.022 \\
\hline C4-C3 & 1.375 & $\mathrm{C} 5-\mathrm{C} 4-\mathrm{C} 3$ & 120.255 & C6-C5-C4-C3 & -179.976 \\
\hline $\mathrm{C} 5-\mathrm{C} 4$ & 1.421 & $\mathrm{C} 6-\mathrm{C} 5-\mathrm{C} 4$ & 122.131 & C7-C6-C5-C4 & -179.955 \\
\hline C6-C5 & 1.420 & C7-C6-C5 & 121.089 & C8-C7-C6-C5 & 0.048 \\
\hline $\mathrm{C} 7-\mathrm{C} 6$ & 1.376 & C8-C7-C6 & 120.320 & C9-C8-C7-C6 & -0.092 \\
\hline $\mathrm{C} 8-\mathrm{C} 7$ & 1.415 & $\mathrm{C9}-\mathrm{C} 8-\mathrm{C} 7$ & 120.296 & C10-C9-C8-C7 & 0.054 \\
\hline C9-C8 & 1.377 & $\mathrm{C} 10-\mathrm{C} 9-\mathrm{C} 8$ & 120468 & O11-C1-C2-C3 & 179.945 \\
\hline $\mathrm{C} 10-\mathrm{C} 9$ & 1.418 & 011-C1-C2 & 122.792 & H12-C2-C3-C4 & 179.953 \\
\hline O11-C1 & 1.368 & $\mathrm{H} 12-\mathrm{C} 2-\mathrm{C} 3$ & 119.938 & H13-C3-C4-C5 & -179.963 \\
\hline $\mathrm{H} 12-\mathrm{C} 2$ & 1.088 & H13-C3-C4 & 120.253 & $\mathrm{H} 14-\mathrm{C} 4-\mathrm{C} 5-\mathrm{C} 6$ & 0.0186 \\
\hline $\mathrm{H} 13-\mathrm{C} 3$ & 1.086 & $\mathrm{H} 14-\mathrm{C} 4-\mathrm{C} 5$ & 119.064 & H15-C6-C7-C8 & -179.981 \\
\hline $\mathrm{H} 14-\mathrm{C} 4$ & 1.086 & $\mathrm{H} 15-\mathrm{C} 6-\mathrm{C} 7$ & 120.364 & $\mathrm{H} 16-\mathrm{C} 7-\mathrm{C} 8-\mathrm{C} 9$ & 179.952 \\
\hline $\mathrm{H} 15-\mathrm{C} 6$ & 1.087 & $\mathrm{H} 16-\mathrm{C} 7-\mathrm{C} 8$ & 119.674 & $\mathrm{H} 17-\mathrm{C} 8-\mathrm{C} 9-\mathrm{C} 10$ & -179.985 \\
\hline $\mathrm{H} 16-\mathrm{C} 7$ & 1.086 & $\mathrm{H} 17-\mathrm{C} 8-\mathrm{C} 9$ & 119.985 & $\mathrm{H} 18-\mathrm{C} 9-\mathrm{C} 10-\mathrm{C} 1$ & 0.086 \\
\hline $\mathrm{H} 17-\mathrm{C} 8$ & 1.086 & $\mathrm{H} 18-\mathrm{C} 9-\mathrm{C} 10$ & 118.791 & $\mathrm{H} 19-011-\mathrm{C} 1-\mathrm{C} 2$ & -0.096 \\
\hline $\mathrm{H} 18-\mathrm{C} 9$ & 1.084 & $\mathrm{H} 19-011-\mathrm{C} 1$ & 108.727 & & \\
\hline $\mathrm{H} 19-011$ & 0.969 & & & & \\
\hline
\end{tabular}

*for numbering of atom refer Fig. 1

\section{B. Analysis of Vibrations Spectra}

The 51 normal modes of 1-Naphthol are distributed among the symmetry species as $\Gamma_{3 N-6}=35 A^{\prime}$ (in-plane) $+16 A^{\prime \prime}$ (out-of-plane), and in agreement with $C_{S}$ symmetry. All the vibrations were active both in Raman scattering and infrared absorption. In the Raman spectrum the in-plane vibrations $\left(A^{\prime}\right)$ give rise to polarized bands while the out-of-plane ones $\left(A^{\prime \prime}\right)$ to depolarized band. The TED were reported in Table 5.

For visual comparison, the observed and simulated FT-IR and FT-Raman spectra of 1-Naphthol are produced in a common frequency scales in Fig. 2 \& Fig. 3. Root mean square (RMS) values of frequencies were obtained in the study using the following expression, 
Raja et al. : Structure and Vibrational Spectroscopic Studies ...

Table 3. Definition of internal coordinates of 1-Naphthol

\begin{tabular}{|c|c|c|c|}
\hline No (i) & Symbol & Type & Definition \\
\hline \multicolumn{4}{|c|}{ Streching } \\
\hline $1-7$ & $r_{i}$ & $\mathrm{C}-\mathrm{H}$ & $\mathrm{C} 2-\mathrm{H} 11, \mathrm{C} 3-\mathrm{H} 13, \mathrm{C} 4-\mathrm{H} 14, \mathrm{C} 6-\mathrm{H} 15, \mathrm{C} 7-\mathrm{H} 16, \mathrm{C} 8-\mathrm{H} 17, \mathrm{C} 9-\mathrm{H} 18$ \\
\hline 8 & $q_{i}$ & $\mathrm{C}-\mathrm{O}$ & C1-011 \\
\hline 9 & $Q_{i}$ & $\mathrm{O}-\mathrm{H}$ & O11-H19 \\
\hline $10-20$ & $R i$ & $\mathrm{C}-\mathrm{C}$ & $\begin{array}{l}\mathrm{C} 1-\mathrm{C} 2, \mathrm{C} 2-\mathrm{C} 3, \mathrm{C} 3-\mathrm{C} 4, \mathrm{C} 4-\mathrm{C} 5, \mathrm{C} 5-\mathrm{C} 6, \mathrm{C} 6-\mathrm{C} 7, \mathrm{C} 7-\mathrm{C} 8, \mathrm{C} 8-\mathrm{C} 9 \\
\mathrm{C} 9-\mathrm{C} 10, \mathrm{C} 10-\mathrm{C} 1, \mathrm{C} 10-\mathrm{C} 5\end{array}$ \\
\hline \multicolumn{4}{|l|}{ Bending } \\
\hline $21-34$ & $\beta_{i}$ & $\mathrm{C}-\mathrm{C}-\mathrm{H}$ & $\begin{array}{l}\text { C1-C2-H12, C3-C2-H12, C2-C3-H13. C4-C3-H13, C3-C4-H14, } \\
\text { C5-C4-H14, C5-C6-H15, C7-C6-H15, C6-C7-H16, C8-C7-H16, } \\
\text { C7-C8-H17, C9-C8-H17, C8-C9-H18, C10-C9-H18. }\end{array}$ \\
\hline $35-36$ & $\theta_{i}$ & $\mathrm{C}-\mathrm{C}-\mathrm{O}$ & C10-C1-011, C2-C1-011 \\
\hline 37 & $\phi i$ & $\mathrm{C}-\mathrm{O}-\mathrm{H}$ & C1-011-H19 \\
\hline $38-43$ & $\alpha_{i}$ & bring 1 & $\begin{array}{l}\text { C1-C2-C3, C2-C3-C4, C3-C4-C5, C4-C5-C10, C5-C10-C5, } \\
\text { C10-C1-C2 }\end{array}$ \\
\hline $44-49$ & $\alpha_{i}$ & bring 2 & $\begin{array}{l}\mathrm{C5}-\mathrm{C} 6-\mathrm{C} 7, \mathrm{C} 6-\mathrm{C} 7-\mathrm{C} 8, \mathrm{C} 7-\mathrm{C} 8-\mathrm{C} 9, \mathrm{C} 8-\mathrm{C} 9-\mathrm{C} 10, \mathrm{C} 9-\mathrm{C} 10-\mathrm{C} 5 \\
\mathrm{C} 10-\mathrm{C} 5-\mathrm{C} 6\end{array}$ \\
\hline \multicolumn{4}{|c|}{ Out-of-plane bending } \\
\hline $50-56$ & $\omega_{i}$ & $\omega \mathrm{C}-\mathrm{H}$ & $\begin{array}{l}\mathrm{H} 12-\mathrm{C} 2-\mathrm{C} 1-\mathrm{C} 3, \mathrm{H} 13-\mathrm{C} 3-\mathrm{C} 2-\mathrm{C} 4, \mathrm{H} 14-\mathrm{C} 4-\mathrm{C} 3-\mathrm{C} 5, \mathrm{H} 15-\mathrm{C} 6-\mathrm{C} 5-\mathrm{C} 7 \\
\mathrm{H} 16-\mathrm{C} 7-\mathrm{C} 6-\mathrm{C} 8, \mathrm{H} 17-\mathrm{C} 8-\mathrm{C} 7-\mathrm{C} 9, \mathrm{H} 18-\mathrm{C} 9-\mathrm{C} 8-\mathrm{C} 10 .\end{array}$ \\
\hline 57 & $\omega_{i}$ & $\omega \mathrm{C}-\mathrm{O}$ & O11-C1-C10-C2 \\
\hline \multicolumn{4}{|l|}{ Torison } \\
\hline $58-63$ & $\tau_{i}$ & tring 1 & $\begin{array}{l}\text { C1-C2-C3-C4, C2-C3-C4-C5, C3-C4-C5-C10, C4-C5-C10-C1, } \\
\text { C5-C10-C1-C2, C10-C1-C2-C3 }\end{array}$ \\
\hline $64-69$ & $\tau_{i}$ & tring 2 & $\begin{array}{l}\text { C5-C6-C7-C8, C6-C7-C8-C9, C7-C8-C9-C10, C8-C9-C10-C5, } \\
\text { C9-C10-C5-C6, C10-C5-C6-C7 }\end{array}$ \\
\hline 70 & $\tau_{i}$ & $\tau \mathrm{O}-\mathrm{H}$ & C2 (C10)-C1-O11-H19 \\
\hline $71-72$ & $\tau_{i}$ & Butterfly & C4-C5-C10-C9, C6-C5-C10-C1 \\
\hline
\end{tabular}

*for numbering of atom refer Fig. 1 
Table 4. Definiton of local symmetry coordinates and the value corresponding scale factors used to correct the force fields for 1-Naphthol

\begin{tabular}{|c|c|c|}
\hline No.(i) & Symbol $^{\mathrm{a}}$ & Definition $^{b}$ \\
\hline $1-7$ & $\mathrm{C}-\mathrm{H}$ & $r 1, r 2, r 3, r 4, r 5, r 6, r 7$ \\
\hline 8 & $\mathrm{C}-\mathrm{O}$ & q8 \\
\hline 9 & $\mathrm{O}-\mathrm{H}$ & Q9 \\
\hline $10-20$ & $\mathrm{C}-\mathrm{C}$ & R10, R11, R12, R13, R14, R15, R16, R17, R18, R19, R20 \\
\hline $21-27$ & $\mathrm{C}-\mathrm{C}-\mathrm{H}$ & $\begin{array}{l}(\beta 21-\beta 22) / \sqrt{2},(\beta 23-\beta 24) / \sqrt{2},(\beta 25-\beta 26) / \sqrt{2}(\beta 27-\beta 28) / \sqrt{2},(\beta \\
29-\beta 30) / \sqrt{2},(\beta 31-\beta 32) / \sqrt{2},(\beta 33-\beta 34) / \sqrt{2}\end{array}$ \\
\hline 28 & C-C-O & $\left(\begin{array}{llll}\theta & 35 & -\theta & 36\end{array}\right) / \sqrt{2}$ \\
\hline 29 & $\mathrm{C}-\mathrm{O}-\mathrm{H}$ & $\phi 37$ \\
\hline 30 & bring 1 & $(\alpha 38-\alpha 39+\alpha 40-\alpha 41+\alpha 42-\alpha 43) / \sqrt{6}$ \\
\hline 31 & bring 1 & $(2 \alpha 38-\alpha 39-\alpha 40+2 \alpha 41-\alpha 42-\alpha 43) \sqrt{12}$ \\
\hline 32 & bring 1 & $(\alpha 39-\alpha 40+\alpha 42-\alpha 43) / 2$ \\
\hline 33 & bring 2 & $\{(\alpha 44-\alpha 45+\alpha 46-\alpha 47+\alpha 48-\alpha 49)\}$ \\
\hline 34 & bring 2 & $(2 \alpha 44-\alpha 45-\alpha 46+2 \alpha 47-\alpha 48-\alpha 49) \sqrt{12}$ \\
\hline 35 & bring 2 & $(\alpha 45-\alpha 46+\alpha 48-\alpha 49) / 2$ \\
\hline $36-42$ & $\omega \mathrm{C}-\mathrm{H}$ & $\omega 50, \omega 51, \omega 52, \omega 53, \omega 54, \omega 55, \omega 56$ \\
\hline 43 & $\omega \mathrm{C}-\mathrm{O}$ & $\omega 57$ \\
\hline 44 & tring 1 & $(\tau 58-\tau 59+\tau 60-\tau 61+\tau 62-\tau 63) \sqrt{6}$ \\
\hline 45 & tring 1 & $(\tau 58-\tau 60+\tau 61-\tau 63) / 2$ \\
\hline 46 & tring 1 & $(-\tau 58+2 \tau 59-\tau 60-\tau 61+2 \operatorname{tal} 62-\tau 63) \sqrt{12}$ \\
\hline 47 & tring 2 & $(\tau 64-\tau 65+\tau 66-\tau 67+\tau 68-\tau 69) \sqrt{6}$ \\
\hline 48 & tring 2 & $(\tau 64-\tau 65+\tau 66-\tau 67) \sqrt{2}$ \\
\hline 49 & tring 2 & $(-\tau 64+2 \tau 65-\tau 66-\tau 67+2 \operatorname{tal} 68-\tau 69) \sqrt{12}$ \\
\hline 50 & tO-H & $\mathrm{t} 70$ \\
\hline 51 & Butterfly & $(\tau 71-\tau 72) / \sqrt{2}$ \\
\hline
\end{tabular}

${ }^{\mathrm{a}}$ These symbols are used for description of the normal modes by TED in Table 5.

${ }^{\mathrm{b}}$ The internal coordinates used here are defined in Table 3. 
Raja et al. : Structure and Vibrational Spectroscopic Studies ...

Table 5. Detailed assignments of fundamental vibrations of 1-Naphthol by normal mode analysis based on SQM force field calculation

\begin{tabular}{|c|c|c|c|c|c|c|c|c|}
\hline \multirow[t]{2}{*}{ No. } & \multirow[t]{2}{*}{$\begin{array}{l}\text { Symmetry } \\
\text { species } \\
\text { Cs }\end{array}$} & \multicolumn{3}{|c|}{$\begin{array}{l}\text { Observed frequency } \\
\qquad\left(\mathrm{cm}^{-1}\right)\end{array}$} & \multicolumn{3}{|c|}{$\begin{array}{l}\text { Calculated frequency } \\
\left(\mathrm{cm}^{-1}\right) \text { with } \\
\text { B3LYP/6-311+G }{ }^{\star *} \text { force } \\
\text { field }\end{array}$} & \multirow[t]{2}{*}{$\begin{array}{c}\text { TED (\%) among type of internal } \\
\text { coordinates }^{c}\end{array}$} \\
\hline & & Infrared & Raman & Unscaled & Scaled & $I R^{a} A_{i}$ & $\operatorname{Raman}^{b} l_{i}$ & \\
\hline 1 & $A^{\prime}$ & 3781 & & 4129 & 3754 & 46.960 & 149.807 & $\mathrm{OH}(100)$ \\
\hline 2 & $A^{\prime}$ & 3258 & & 3549 & 3227 & 8.568 & 128.455 & $\mathrm{CH}(99)$ \\
\hline 3 & $\overline{A^{\prime}}$ & & & 3526 & 3206 & 20.078 & 329.074 & $\mathrm{CH}(99)$ \\
\hline 4 & $A^{\prime}$ & & & 3524 & 3204 & 39.605 & 86.932 & $\mathrm{CH}(99)$ \\
\hline 5 & $\overline{A^{\prime}}$ & 3222 & & 3510 & 3191 & 20.519 & 126.357 & $\mathrm{CH}(99)$ \\
\hline 6 & $A^{\prime}$ & 3218 & & 3505 & 3187 & 4.776 & 58.963 & $\mathrm{CH}(99)$ \\
\hline 7 & $A^{\prime}$ & & & 3495 & 3178 & 1.212 & 39.227 & $\mathrm{CH}(99)$ \\
\hline 8 & $A^{\prime}$ & 3198 & & 3483 & 3167 & 20.719 & 106.842 & $\mathrm{CH}(99)$ \\
\hline 9 & $\overline{A^{\prime}}$ & 1705 & 1681 & 1857 & 1689 & 12.219 & 3.569 & $\begin{array}{l}\mathrm{CC}(66), \text { bCH (13), bring } 1 \text { (9), } \\
\text { bring } 2 \text { (8) }\end{array}$ \\
\hline 10 & $A^{\prime}$ & & & 1823 & 1658 & 28.033 & 0.697 & $\mathrm{CC}(68), \mathrm{bCH}(23)$ \\
\hline 11 & $A^{\prime}$ & 1652 & & 1799 & 1636 & 51.772 & 47.257 & $\begin{array}{l}\mathrm{CC}(69), \text { bCH (13), bring } 1 \text { (8), } \\
\text { bring } 2(6)\end{array}$ \\
\hline 12 & $A^{\prime}$ & 1588 & 1583 & 1730 & 1573 & 13.244 & 0.730 & $\mathrm{CC}(57), \mathrm{bCH}(32)$ \\
\hline 13 & $A^{\prime}$ & 1529 & 1519 & 1666 & 1515 & 7.486 & 14.639 & $\mathrm{bCH}(44), \mathrm{CC}(41)$ \\
\hline 14 & $A^{\prime}$ & 1519 & & 1655 & 1505 & 7.114 & 51.206 & $\mathrm{bCH}(59), \mathrm{CC}(33)$ \\
\hline 15 & $A^{\prime}$ & 1462 & 1461 & 1592 & 1448 & 18.458 & 2.200 & $\begin{array}{l}\mathrm{bCH}(59), \mathrm{CC}(25), \text { bring } 1 \text { (7), } \mathrm{CO} \\
(6)\end{array}$ \\
\hline 16 & $A^{\prime}$ & 1443 & & 1571 & 1429 & 45.380 & 96.795 & $\mathrm{CC}(78), \mathrm{bCH}(12)$ \\
\hline 17 & $A^{\prime}$ & 1420 & & 1547 & 1407 & 13.142 & 55.089 & $\mathrm{CC}(79), \mathrm{bCH}(12)$ \\
\hline 18 & $\overline{A^{\prime}}$ & 1332 & 1312 & 1450 & 1319 & 78.985 & 5.138 & $\begin{array}{l}\mathrm{bCH}(36), \mathrm{CO}(27), \mathrm{CC}(21), \text { bring } \\
1(15)\end{array}$ \\
\hline 19 & $A^{\prime \prime}$ & 1294 & 1281 & 1410 & 1282 & 25.568 & 2.207 & $\begin{array}{l}\mathrm{bCH}(52), \mathrm{CC}(28), \text { bring } 1 \text { (8), CO } \\
(6)\end{array}$ \\
\hline 20 & $A^{\prime}$ & 1281 & 1271 & 1395 & 1269 & 36.671 & 4.786 & $\begin{array}{l}\mathrm{CC}(33), \mathrm{bOH}(28), \mathrm{bCH}(25), \text { bring } \\
2 \text { (12) }\end{array}$ \\
\hline 21 & $A^{\prime}$ & 1245 & 1242 & 1356 & 1233 & 30.830 & 6.080 & $\mathrm{CC}(48), \mathrm{bCH}(31), \mathrm{bOH}(19)$ \\
\hline 22 & $A^{\prime}$ & 1208 & 1209 & 1316 & 1197 & 3.895 & 3.187 & $\mathrm{bCH}(76), \mathrm{CC}(14)$ \\
\hline 23 & $A^{\prime}$ & 1198 & & 1305 & 1187 & 14.717 & 1.073 & $\mathrm{bCH}(59), \mathrm{CC}(35), \mathrm{bOH}(5)$ \\
\hline 24 & $\overline{A^{\prime}}$ & 1187 & 1184 & 1293 & 1176 & 9.480 & 8.844 & $\begin{array}{l}\mathrm{bCH}(52), \mathrm{CC}(33), \text { bring } 1 \text { (8), } \\
\mathrm{bOH}(6)\end{array}$ \\
\hline
\end{tabular}




\begin{tabular}{|c|c|c|c|c|c|c|c|c|}
\hline \multirow[t]{2}{*}{ No. } & \multirow[t]{2}{*}{$\begin{array}{l}\text { Symmetry } \\
\text { species } \\
\text { Cs }\end{array}$} & \multicolumn{3}{|c|}{$\begin{array}{l}\text { Observed frequency } \\
\qquad\left(\mathrm{cm}^{-1}\right)\end{array}$} & \multicolumn{3}{|c|}{$\begin{array}{l}\text { Calculated frequency } \\
\left(\mathrm{cm}^{-1}\right) \text { with } \\
\text { B3LYP/6-311+G force } \\
\text { field }\end{array}$} & \multirow[t]{2}{*}{$\begin{array}{l}\text { TED (\%) among type of internal } \\
\text { coordinates }\end{array}$} \\
\hline & & Infrared & Raman & Unscaled & Scaled & $I R^{a} A_{i}$ & $\operatorname{Raman}^{\mathrm{b}} l_{i}$ & \\
\hline 25 & $A^{\prime}$ & 1121 & 1148 & 1222 & 1111 & 15.005 & 7.658 & $\begin{array}{l}\text { CC (45), bCH (27), bring } 2 \text { (17), } \\
\mathrm{CO}(8)\end{array}$ \\
\hline 26 & $A^{\prime}$ & 1081 & 1086 & 1178 & 1071 & 39.447 & 6.243 & $\begin{array}{l}\mathrm{CC}(37), \text { bring } 2(19), \mathrm{bCH}(16), \\
\mathrm{CO}(10), \text { bring } 1(8)\end{array}$ \\
\hline 27 & $A^{\prime}$ & 1059 & 1045 & 1153 & 1049 & 15.865 & 7.558 & $\mathrm{CC}(74), \mathrm{bCH}(14)$ \\
\hline 28 & $A^{\prime \prime}$ & 1001 & & 1091 & 992 & 0.002 & 0.103 & gCH (87), tring 2 (13) \\
\hline 29 & $A^{\prime \prime}$ & 973 & & 1060 & 964 & 1.786 & 0.044 & $\mathrm{~g} \mathrm{CH} \mathrm{(90)}$ \\
\hline 30 & $A^{\prime \prime}$ & 964 & 960 & 1050 & 955 & 0.027 & 0.027 & $\mathrm{gCH}(92)$, tring 1 (6) \\
\hline 31 & $A^{\prime}$ & 899 & & 980 & 891 & 10.449 & 3.571 & $\begin{array}{l}\text { bring } 1 \text { (34), bring } 2 \text { (33), CC (20), } \\
\text { CO (7) }\end{array}$ \\
\hline 32 & $A^{\prime \prime}$ & 896 & 877 & 976 & 888 & 1.298 & 4.247 & $\mathrm{gCH}(75)$, tring 2 (14), tring $1(10)$ \\
\hline 33 & $A^{\prime \prime}$ & 862 & 861 & 939 & 854 & 0.002 & 1.367 & $\mathrm{gCH}(81)$, tring 1 (11), gCO (7) \\
\hline 34 & $A^{\prime \prime}$ & 810 & & 883 & 803 & 41.362 & 2.603 & $\begin{array}{l}\text { gCH (40), tring } 1(31) \text {, tring } 2(20) \text {, } \\
\mathrm{gCO}(9)\end{array}$ \\
\hline 35 & $\overline{A^{\prime}}$ & 808 & & 881 & 801 & 6.361 & 0.558 & bring 1 (40), bring 2 (33), CC (23) \\
\hline 36 & $A^{\prime \prime}$ & 796 & 790 & 867 & 789 & 54.610 & 1.294 & $\begin{array}{l}\mathrm{gCH}(59), \text { tring } 2(16), \text { tring } 1(15), \\
\mathrm{gCO}(6)\end{array}$ \\
\hline 37 & $A^{\prime \prime}$ & 752 & 772 & 819 & 745 & 1.880 & 5.893 & $\mathrm{gCH}(74)$, tring $2(19)$, tring $1(7)$ \\
\hline 38 & $A^{\prime}$ & 733 & 716 & 798 & 726 & 3.145 & 29.302 & $\begin{array}{l}\text { CC (48), bring } 2(31), \text { bring } 1(8) \text {, } \\
\text { CO (8) }\end{array}$ \\
\hline 39 & $A^{\prime}$ & 652 & 630 & 710 & 646 & 1.071 & 29.302 & $\begin{array}{l}\text { tring } 2 \text { (33), gCO (26), tring } 1 \text { (25), } \\
\mathrm{gCH}(14)\end{array}$ \\
\hline 40 & $A^{\prime}$ & 590 & 580 & 643 & 585 & 3.438 & 1.666 & bring 2 (50), bring 1 (24), CC (16) \\
\hline 41 & $A^{\prime \prime}$ & 590 & 575 & 643 & 585 & 1.185 & 8.756 & $\begin{array}{l}\text { tring } 1 \text { (43), gCH (19), tring } 2 \text { (14), } \\
\text { tCC (12), gCO (12) } \\
\end{array}$ \\
\hline 42 & $A^{\prime}$ & 538 & 540 & 586 & 533 & 4.871 & 4.995 & $\begin{array}{l}\text { bring } 1 \text { (34), bring } 2 \text { (30), bCO } \\
\text { (19), CC (13) }\end{array}$ \\
\hline 43 & $A^{\prime}$ & 491 & 485 & 535 & 487 & 0.846 & 5.765 & bring 1 (59), bring 2 (24), CC (11) \\
\hline 44 & $A^{\prime \prime}$ & 483 & & 526 & 479 & 0.389 & 0.344 & tring 1 (42), tring 2 (40), gCH (15) \\
\hline 45 & $A^{\prime}$ & 474 & 467 & 517 & 470 & 0.581 & 5.884 & $\begin{array}{l}\text { bCO (37), CC (25), bring } 1 \text { (19), } \\
\text { bring } 2 \text { (14) }\end{array}$ \\
\hline 46 & $A^{\prime \prime}$ & 437 & & 476 & 433 & 0.395 & 1.787 & $\begin{array}{l}\text { tring } 2(56), \text { gCH (16), tCC (10), } \\
\text { gCO (9), tring } 1(5)\end{array}$ \\
\hline 47 & $A^{\prime \prime}$ & 371 & & 404 & 368 & 113.069 & 5.174 & $\mathrm{tOH}(84)$, tring $2(10)$ \\
\hline 48 & $\overline{A^{\prime}}$ & 286 & 283 & 312 & 284 & 6.294 & 1.346 & $\begin{array}{l}\text { bCO (38), bring } 2 \text { (25), bring } 1 \\
(21), \text { CC (14) }\end{array}$ \\
\hline
\end{tabular}




\begin{tabular}{|c|c|c|c|c|c|c|c|c|}
\hline \multirow[t]{2}{*}{ No. } & \multirow[t]{2}{*}{$\begin{array}{l}\text { Symmetry } \\
\text { species } \\
\text { Cs }\end{array}$} & \multicolumn{3}{|c|}{$\begin{array}{l}\text { Observed frequency } \\
\qquad\left(\mathrm{cm}^{-1}\right)\end{array}$} & \multicolumn{3}{|c|}{$\begin{array}{l}\text { Calculated frequency } \\
\left(\mathrm{cm}^{-1}\right) \text { with } \\
\text { B3LYP/6-311+G } \text { Gorce }^{\star *} \text { field }\end{array}$} & \multirow[t]{2}{*}{$\begin{array}{c}\text { TED (\%) among type of interna } \\
\text { coordinates }\end{array}$} \\
\hline & & Infrared & Raman & Unscaled & Scaled & $I R^{a} A_{i}$ & $\operatorname{Raman}^{\mathrm{b}} l_{i}$ & \\
\hline 49 & $A^{\prime \prime}$ & 267 & & 291 & 265 & 4.465 & 1.014 & $\begin{array}{l}\text { tring } 1 \text { (45), tring } 2 \text { (21), gCH (15), } \\
\text { tCC (11), gCO (7) }\end{array}$ \\
\hline 50 & $A^{\prime \prime}$ & 179 & 171 & 195 & 178 & 4.019 & 0.026 & $\begin{array}{l}\text { tCC (28), tring } 2 \text { (25), tring } 1 \text { (25), } \\
\mathrm{gCH}(16), \mathrm{gCO}(6)\end{array}$ \\
\hline 51 & $A^{\prime \prime}$ & 144 & & 157 & 143 & 0.457 & 2.428 & tring $1(60)$, tring $2(31), \mathrm{gCH}(7)$ \\
\hline
\end{tabular}

Abbreviations used: $b$, bending; $g$, wagging; $t$, torsion;

${ }^{a}$ Relative absorption intensities normalized with highest peak absorption

${ }^{b}$ Relative Raman intensities calculated by Eq.1 and normalized to 100 .

${ }^{\mathrm{C}}$ For the notations used see Table 4.

$$
R M S=\sqrt{\frac{1}{n-1} \sum_{n-1}^{1}\left(U_{1}^{\text {calc }}-U_{1}^{\exp }\right)^{2}}
$$

The RMS error of the observed and calculated frequencies (unscaled / B3LYP/6-311+ $G^{* *}$ ) of 1-Naphthol was found to be $107 \mathrm{~cm}^{-1}$. This is quite obvious; since the frequencies calculated on the basis of quantum mechanical force fields usually differ

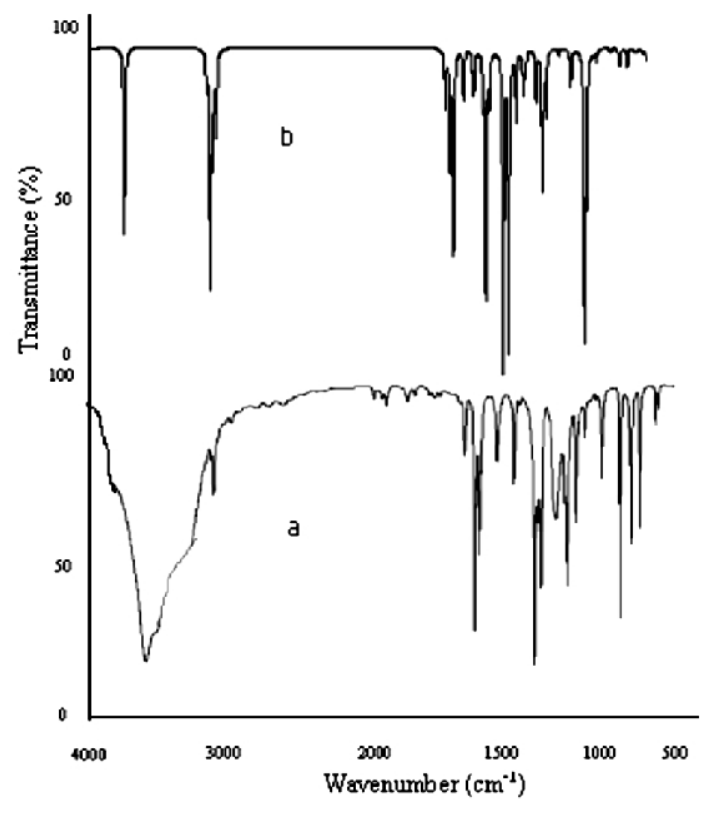

Fig. 2 FT-IR spectra of 1-Naphthol.

(a) Observed (b) Calculated with

$$
B 3 L Y P / 6-311+G^{* *}
$$

appreciably from observed frequencies. This is partly due to the neglect of anharmonicity and partly due to the approximate nature of the quantum mechanical methods. In order to reduce the overall deviation between the unscaled and observed fundamental frequencies, scale factors were applied in the normal coordinate analysis and the subsequent least square fit refinement algorithm resulted into a very close agreement between the observed fundamentals and the scaled frequencies. Refinement of the scaling factors applied in this study achieved a weighted mean

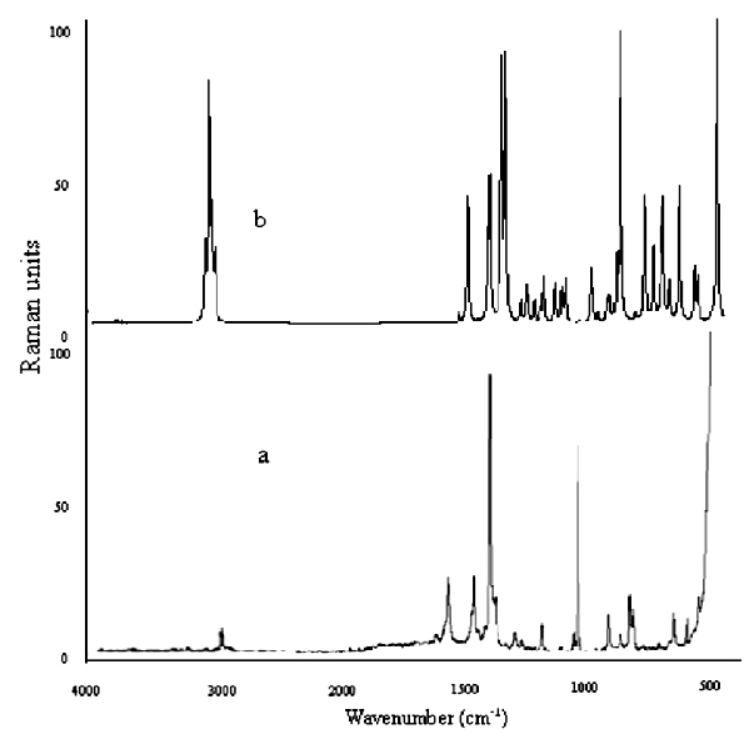

Fig. 3 FT-Raman spectra of 1-Naphthol.

(a) Observed (b) Calculated with

$$
B 3 L Y P / 6-311+G^{* *}
$$


deviation of $9 \mathrm{~cm}^{-1}$ between the experimental and scaled frequencies of the title compound.

\section{C-C vibrations}

The bands between 1407, 1636 and $1658 \mathrm{~cm}^{-1}$ are assigned to $\mathrm{C}-\mathrm{C}$ stretching modes [24]. In the present study, the carbon stretching vibrations of the title compound have been observed at $1420,1652 \mathrm{~cm}^{-1}$ in the FT-IR and $1583 \mathrm{~cm}^{-1}$ in FT-Raman spectrum and are presented in Table 5. These assignments are in good agreement with literature $[25,26]$. In present investigation, the $\mathrm{C}-\mathrm{C}$ mode mixes with $\mathrm{C}-\mathrm{H}$ in-plane bending vibrations.

\section{$\mathrm{C}-\mathrm{H}$ vibrations}

The presence of hetro-aromatic-type structure is best recognized by the presence of $\mathrm{C}-\mathrm{H}$ stretching vibrations [27] near $3200 \mathrm{~cm}^{-1}$. Aromatic compounds commonly exhibit multiple weak bands in the region 3100-3000 $\mathrm{cm}^{-1}$ due to aromatic $\mathrm{C}-\mathrm{H}$ stretching vibrations. The bands due to $\mathrm{C}-\mathrm{H}$ in-plane ring bending vibration interacting with $\mathrm{C}-\mathrm{C}$ stretching vibration are observed as a number of $\mathrm{m}-\mathrm{w}$ intensity sharp bands in the region $1300-1000 \mathrm{~cm}^{-1}$. C-H out-of-plane bending vibrations are strongly coupled vibrations and occur in the region $900-667 \mathrm{~cm}^{-1}$. Accordingly, in the present study the $\mathrm{C}-\mathrm{H}$ vibrations of the title compounds are occured at 3227, 3206, 3204, 3191, 3187, 3178 and $3167 \mathrm{~cm}^{-1}$ for 1-Naphthol.

\section{C-O vibrations}

The non-linearity of hydrogen bond in 1-Naphthol have an impact over the carbonyl group frequency. The interaction of carbonyl group with the other group present in the system does not produce such a drastic and characteristic changes in the frequency of $\mathrm{C} \mathrm{O}$ stretch. The carbonyl stretching frequency is very sensitive to the factors that disturb the nature of the carbonyl group and its precise frequency is characteristic of the type of the carbonyl compound being studied. Particularly detailed correlations have been made for the carbonyl bond stretching frequency. The carbonyl stretching frequency has been most extensively studied by infrared spectroscopy. This multiply bonded group is highly polar ( $>C d^{\delta+}=O d^{\delta-}$ ) and therefore gives rise to an intense infrared absorption band. The carbon-oxygen dou-ble bond is formed by the $p p_{\pi}-p p_{\pi}$ bonding between carbon and oxygen. Because of the different electro-negativities of carbon and oxygen atoms, the bonding electrons are not equally distributed between the two atoms. The following two resonance forms contribute to the bonding of the car-bonyl group $>\mathrm{C}=\mathrm{O} \leftrightarrow \mathrm{C}+-\mathrm{O}^{-}$. The lone pair of electrons on oxygen also determines the nature of the carbonyl group. The position of the $\mathrm{C}=\mathrm{O}$ stretching vibration is very sensi-tive to various factors such as the physical state, electronic effects by substituents, ring strains, etc. [24]. Consideration of these factors provides further information about the environ-ment of the $\mathrm{C}=\mathrm{O}$ group. The carbonyl stretching generally occurs as a strong absorption in the region from 1448, 1319, 1282, 1111, $1071,891 \mathrm{~cm}^{-1}$. This portion of the infrared spectrum is most useful because the position of the carbonyl absorption is quite sensitive to substitution effects and the geometry of the molecule.

\section{$\mathrm{OH}$ vibrations}

The precise positions of $\mathrm{O}-\mathrm{H}$ band are dependent on the strength of hydrogen bond. The $\mathrm{O}-\mathrm{H}$ stretching appears at $3800-3500 \mathrm{~cm}^{-1}$ in the inter-molecular hydrogen bonded systems. The observed peaks in this region are sharp and strong. The title compounds in this study showed a very strong absorption peak at $3754 \mathrm{~cm}^{-1}$ which are due to the $\mathrm{O}-\mathrm{H}$ stretching vibrations.

\section{$\mathrm{C}-\mathrm{O}$ stretching and $\mathrm{O}-\mathrm{H}$ bending vibrations}

Two bands arising from $\mathrm{C}-\mathrm{O}$ stretching and $\mathrm{O}-\mathrm{H}$ bending appear in the spectra of carboxylic acids near 1210-1320 and 1400-1440 $\mathrm{cm}^{-1}$, respectively. Both these bands involve some interaction between $\mathrm{C}-\mathrm{O}$ stretching and in-plane $\mathrm{C}-\mathrm{O}-\mathrm{H}$ bending. The more intense band near $1280-1315 \mathrm{~cm}^{-1}$ for dimers is generally referred to as $\mathrm{CO}$ stretching band and it usually occurs as a doublet in the spectra of long-chain fatty acids. One of the characteristic bands in the spectra of dimeric carboxylic acid arises from the out-of-plane bending of the hydrogen bonded $\mathrm{OH}$. It appears near $1071 \mathrm{~cm}^{-1}$ and is characteristically broad with medium intensity [24].

\section{Ring vibrations}

Several ring modes are affected by the substitution in the aro-matic ring. In the present study, the bands absorbed at 1689, 1636, 1448, 1319, 1282 $\mathrm{cm}^{-1}$ and $891,801,585,487 \mathrm{~cm}^{-1}$ have been designated to ring in-plane and out-of-plane bending modes, respectively. For most of the remaining ring 
vibrations, the overall agreement is satisfactory. Small changes in frequencies observed for these modes are due to the changes in force constants/reduced mass ratio resulting mainly due to the extent of mixing between ring and substituent group.

\section{Hyperpolarizability calculations}

The first-order hyperpolarizibility $\left(\beta_{i j k}\right)$ of the novel molecular system of 1-Naphthol is calculated using 3-21 $G(d, p)$ basis set based on finite field approach. Hyperpolarizibility is a third rank tensor that can be described by a $3 \times 3 \times 3$ matrix. It strongly depends on the method and basis set used. The 27 components of $3 \mathrm{D}$ matrix can be reduced to 10 components due to Kleinman symmetry [28]. The calculated first-order hyperpolarizability (btotal) of 1-Naphthol is $1.1070 \times 10-30^{-30}$ esu, which is nearly six times that of urea $\left(0.1947 \times 10^{-30}\right.$ esu). The calculated dipole moment $(\mu)$ and first-order hyperpolarizibility $(\beta)$ are shown in Table 6 . The theoretical calculation seems to be more helpful in determination of particular compo-nents of $\beta$ tensor than in establishing the real values of $\beta$. Domination of particular components indicates on a substantial delocalization of charges in those directions. It is noticed that in $\beta_{y y y}$ (which is the principal dipole moment axis and it is parallel to the charge transfer axis) direction, the biggest values of hyperpolarizability are noticed and subsequently delocalization of electron cloud is more in that direction. The higher dipole moment values are associated, in general, with even larger projection of $\beta_{\text {total }}$ quantities. The electric dipoles may enhance, oppose or at least bring the dipoles out of the required net alignment necessary for NLO properties such as $\beta_{\text {total }}$ total values. The connection between the electric dipole mo-ments of an organic molecule having donor-acceptor substituent and first hyperpolarizability is widely recog-nized in the literature [29]. The maximum $\beta$ was due to the behavior of non-zero $\mu$ value. One of the conclusions obtained from this work is that non-zero $\mu$ value may enable the finding of a non-zero $\beta$ value. Of course Hartee-Fock calculations depend on the mathematical method and basis set used for a polyatomic molecule.
Table 6. The dipole moment $(\mathrm{m})$ and First-order hyperpolarizibility (b) of 1-Naphthol derived from dft calculations

\begin{tabular}{|c|c|}
\hline$\beta_{x x x}$ & -30.036823 \\
\hline$\beta_{x x y}$ & 30.0437602 \\
\hline$\beta_{x y y}$ & -13.7476317 \\
\hline$\beta_{y y y}$ & -161.8470719 \\
\hline$\beta_{z x x}$ & 0.0600745 \\
\hline$\beta_{x y z}$ & 0.1220257 \\
\hline$\beta_{z y y}$ & -0.6719205 \\
\hline$\beta_{x z z}$ & 0.4894769 \\
\hline$\beta_{y z z}$ & 0.0155964 \\
\hline$\beta_{z z z}$ & -0.0025623 \\
\hline$b_{\text {total }}$ & 1.1070 \\
\hline$\mu_{x}$ & 0.4960665 \\
\hline$\mu_{y}$ & 0.1145269 \\
\hline$\mu_{z}$ & 0.0019529 \\
\hline$\mu$ & 0.5091 \\
\hline
\end{tabular}

Dipole moment $(\mu)$ in Debye, hyperpolarizibility $\beta(-2 \omega ; \omega, \omega) 10^{-30}$ esu.

\section{CONCLUSION}

In this work, the SQM force field method based on DFT calculations at the B3LYP/6 $-311+G^{* *}$ level have been carried out to analyze the vibrational frequencies of 1-Naphthol. Refinement of the scaling factors applied in this study achieved a weighted RMS deviation of $9 \mathrm{~cm}^{-1}$ between the experimental and scaled frequencies. This close agreement established between the experimental and scaled frequencies obtained using large basis set $\left(6-311+G^{* *}\right)$ calculations has proved to be more reliable and accurate than the calculations using lower basis sets. The first-order hyperpolarizibility $\left(\beta_{i j k}\right)$ of the novel molecular system of 1-Naphthol is calculated using 3-21 G (d,p) basis set based on finite field approach. 
The calculated first-order hyperpolarizability $\left(\beta_{\text {total }}\right)$ of 1-Naphthol is $1.1070 \times 10^{-30}$ esu, which is nearly six times that of urea $\left(0.1947 \times 10^{-30} \mathrm{esu}\right)$.

\section{ACKNOWLEDGEMENT}

The authors are thankful to the Sophisticated Analytical Instrumentation Facility (SAIF), IIT Madras, Chennai, for spectral measurements. Help rendered by S. Anbarasan, Spectroscopic Division, Photonics Research Foundation, Salem, in the calculation part highly acknowledged.

\section{REFERENCES}

[1] B.A. Hess Jr., J. Schaad, P. Carsky, R. Zahradnik, Chem. Rev. 86 (1986) 709.

[2] P. Pulay, X. Zhou, G. Fogarasi, in: R. Fausto (Ed.), NATO ASI Series, vol. C406, Kluwer, Dordrecht, 1993.

[3] C.E. Blom, C. Altona, Mol. Phys. 31 (1976) 1377.

[4] P. Pulay, G. Fogarasi, G. Pongor, J.E. Boggs, A. Vargha, J. Am. Chem. Soc. 105 (1983) 7037.

[5] G. Fogarasi, P. Pulay, in: J.R. Durig (Ed.), Vibrational Spectra and Structure, vol. 14, Elsevier, Amsterdam, 1985.

[6] G. Fogarasi, Spectrochim. Acta 53A (1997) 1211.

[7] G. Pongor, P. Pulay, G. Fogarasi, J.E. Boggs, J. Am. Chem. Soc. 106 (1984) 2765.

[8] G.R. De Mare, Y.N. Panchenko, C.W. Bock, J. Phys. Chem. 98 (1994) 1416.

[9] Y. Yamakita, M. Tasumi, J. Phys. Chem. 99 (1995) 8524.

[10] M.J. Frisch, G.W. Trucks, H.B. Schlega, G.E. Scuseria, M.A. Robb, J.R. Cheesman, V.G. Zakrzewski, J.A. Montgomery Jr., R.E. Stratmann, J.C. Burant, S. Dapprich, J.M. Millam, A.D. Daniels, K.N. Kudin, M.C. Strain, O. Farkas, J. Tomasi, V. Barone, M. Cossi, R. Cammi, B. Mennucci, C. Pomelli, C. Adamo, S. Clifford, J. Ochterski, G.A. Petersson, P.Y. Ayala, Q. Cui, K. Morokuma, N. Roga, P. Salvador, J.J. Dannenberg, D.K. Malick, A.D. Rabuck, K. Rahavachari, J.B. Foresman, J. Cioslowski, J.V. Ortiz, A.G. Baboul, B.B. Stefanov, G. Liu, A. Liashenko, P. Piskorz, I. Komaromi, R. Gomperts, R.L. Martin, D.J. Fox, T. Keith, M.A. Al-Laham, C.Y. Penng, A. Nanayakkara, M. Challa-Combe, P.M.W. Gill, B.
Johnson, W. Chen, M.W. Wong, J.L. Andres, C. Gonzalez, M. Head-Gordon, E.S. Replogle and J.A. Pople, Gaussian 98, Revision A 11.4, Gaussian Inc., Pittsburgh, PA (2002).

[11] A.D. Becke, J.Chem. Phys.98 (1993) 5648.

[12] C. Lee, W. Yang, R.G. Parr, Phys. Rev. B.37 (1998) 785.

[13] P. Pulay, G. Fogarasi, G. Pongor, J.E. Boggs, A. Vargha, J.Am. Chem. Soc. 105 (1983) 7037.

[14] G. Rauhut, P. Pulay, J. Phys. Chem. 99 (1995) 3093.

[15] G. Fogarasi and P. Pulay In: J.R. Durig, Editor, Vibrational Spectra and Structure vol. 14, Elsevier, Amsterdam (1985), p. 125 (Chapter 3).

[16] G. Fogarasi, X. Xhov, P.W. Taylor and P. Pulay, J. Am. Chem. Soc. 114 (1992), p. 8191.

[17] T. Sundius. J. Mol. Struct. 218 (1990) 321.

[18] (a) T. Sundius, Vib. Spectrosc. 29 (2002) 89-95. (b) MOLVIB (v.7.0), Calculation of harmonic force fields and vibrational modes of molecules, QCPE Program No. 807, 2002.

[19] P.L. Polavarapu, J. Phys. Chem. 94 (1990) 8106.

[20] G. Keresztury, S. Holly, J. Varga, G. Besenyei, A.V. Wang, J.R. Durig, Spectrochim. Acta 49A (1993) 2007.

[21] G. Keresztury, in: J.M. Chalmers and P.R. Griffiths (Eds), Handbook of Vibrational Spectroscopy vol.1, John Wiley \& Sons Ltd. (2002), p. 71.

[22] A.D. Becke, J.Chem. Phys. 98 (1993) 5648.

[23] H.D. Cohen, C.C.J. Roothan, J. Chem. Phys. 435 (1965) S34.

[24] D.N. Sathyanarayana, Vibrational Spectroscopy-Theory and Applica-tions, second ed., New Age International (P) Limited Publishers, New Delhi, 2004.

[25] George Socrates, Infrared and Raman Characteristic Group Frequencies -Tables and Charts (third ed.), John Wiley \& Sons, Chichester (2001).

[26] V. Krishna kumar, R. John Xavier, Indian J. Pure Appl. Phys. 41 (2003) 95.

[27] B. Lakshmaiah, G. Ramana Rao, J. Raman Spectrosc. 20 (1989) 439.

[28] D.A. Kleinman, Phys. Rev. 126 (1962) 1977.

[29] P.N. Prasad, D.J. Williams, Introduction to Nonlinear Optical Effects in Molecules and Polymers, Wiley, New York, 1991. 